\title{
Effectiveness and tolerability of ezetimibe co-administered with statins versus statin dose-doubling in high-risk patients with persistent hyperlipidemia: The EZE(STAT)2 trial
}

\author{
A. Shekhar Pandey ${ }^{1}$, Stéphane Bissonnette ${ }^{2}$, Stella Boukas³, Emmanouil Rampakakis ${ }^{3,4}$, \\ John S. Sampalis ${ }^{3,4}$
}

\author{
${ }^{1}$ Cambridge Cardiac Care Centre, Cambridge, Ontario, Canada \\ 2 Merck \& Kirkland, Québec, Canada \\ 3JSS Medical Research Inc., Montréal, Québec, Canada \\ ${ }^{4}$ McGill University, Montréal, Québec, Canada
}

Submitted: 20 July 2010

Accepted: 28 December 2010

Arch Med Sci 2011; 7, 5: 767-775

DOI: 10.5114/aoms.2011.25550

Copyright (c) 2011 Termedia \& Banach

\section{Abstract}

Introduction: When a standard dose of statins fails to achieve lipid control in patients at high risk for coronary artery disease (CAD), increasing the statin dosage or co-administration of additional agents is recommended. The aim of this study was to compare the safety and lipid-lowering efficacy of doubling the standard statin dose (STAT2) to that of co-administering ezetimibe $10 \mathrm{mg} / \mathrm{day}$ (EZE + statin) in Canadian patients at high CAD risk with persistent hyperlipidemia upon statin treatment.

Material and methods: Six-week, open-label, randomized, multicentre study. The primary outcome was the change in plasma LDL-C and secondary measures included the change in additional lipid parameters. Safety was assessed with the incidence of emergent adverse events (AEs).

Results: Eight hundred eighty-five patients (EZE + statin, $n=586$; STAT2, $n=299$ ) completed the study. The mean (SD) percent change in low-density lipoprotein cholesterol (LDL-C) was $-30.9 \%$ (18.2) for the EZE + statin group and $-18.4 \%(19.0)$ for the STAT2 group $(p=0.001)$. Percent and absolute decreases in total cholesterol (TC), triglycerides and the TC to high-density lipoprotein cholesterol ratio (TC/HDL-C) were significantly greater for the EZE + statin group $(p=0.001)$. After 6 weeks of treatment, $70 \%$ of the patients in the EZE + statin group and $48 \%$ of patients in the STAT2 group (OR $=2.45, p<0.001)$ achieved target LDL-C levels of $<2.5 \mathrm{mmol} / \mathrm{l}$. Incidence of AEs was similar between groups, with the exception of a higher incidence of muscle disorders in the STAT2 group. Conclusions: In patients at high CAD risk who are above the LDL-C target while on statin monotherapy, co-administration of ezetimibe is well tolerated and more effective in improving the lipid profile compared to doubling the existing statin dose.

Key words: ezetimibe, hypercholesterolemia, low-density lipoprotein cholesterol, statin.

\section{Introduction}

Cardiovascular disease is the major cause of death globally, accounting for $29 \%$ of all deaths, and its prevalence is expected to increase within the

\author{
Corresponding author: \\ Dr. John S. Sampalis \\ 4492 St. Catherine St. West \\ Westmount, Quebec \\ H3Z 1R7, Canada \\ Phone: (514) 934-6116, \\ ext. 232 \\ Fax: (514) 934-9913 \\ E-mail: \\ jsampalis@jssresearch.com
}


next 2 decades [1]. The importance of hypercholesterolemia in increasing the risk of developing cardiovascular artery disease (CAD) has been well documented by numerous epidemiological studies $[2,3]$. Currently, the gold standard for treating CAD involves modifications in lifestyle and pharmacological intervention with statins to reduce the lowdensity lipoprotein cholesterol (LDL-C). For a large number of patients, however, statin monotherapy is not effective in achieving target LDL-C levels [4-6]. For these patients, titration to higher statin doses or co-administration of additional complementary lipidlowering agents is recommended by the Canadian Cardiovascular Society [7].

Ezetimibe is a cholesterol absorption inhibitor which interferes with the uptake of dietary and biliary cholesterol from the small intestine [8]. Coadministration of ezetimibe with statins results in a greater reduction of LDL-C levels and enhanced improvement of the lipid profile compared with statin monotherapy [9-14], while protecting against the risk of adverse events when using high-dose statins [15-17].

The purpose of this open-label randomized trial was to compare, in a real-life setting, the effectiveness and tolerability of ezetimibe $10 \mathrm{mg} /$ day coadministered with the existing statin regimen versus doubling of the current statin dose in patients at high CAD risk who had not achieved target LDL-C levels while on statin monotherapy.

\section{Material and methods}

\section{Patient population}

Male and female adults with a diagnosis of primary hypercholesterolemia, who were at high risk for coronary artery disease (as defined by a Framingham 10-year risk for $C A D \geq 20 \%$, or medical history of diabetes mellitus and/or atherosclerosis disease) and had LDL-C $\geq 2.5 \mathrm{mmol} / /$ while on statin treatment, were eligible for the study [18]. Additional inclusion criteria included a stable medication regimen and a stable diet for at least 4 weeks prior to study screening. Patients were excluded if they were treated with any other investigational drug within 30 days prior to study recruitment, had any clinically significant concomitant disease which would render them unable to complete the study or place them at risk, were treated with any medication that might interact negatively with statins or ezetimibe or affect the patient's serum lipid levels within eight weeks, or experienced myocardial infarction or coronary intervention within 3 months. Use of cardiovascular medication was allowed provided that the dose was stable for at least 6 weeks prior to study entry and the duration of the study. Hormone replacement therapy in women was also allowed at a stable dose for at least 8 weeks prior to the screening visit and during the study.

\section{Study design}

This was a 6-week, prospective, randomized, open-label trial on patients recruited from the practices of 241 Canadian general practitioners. Eligible patients signed informed consent prior to study enrolment. The study was approved by two independent ethics review boards (IRB services in Aurora, Ontario, Canada; College of Physicians and Surgeons of Alberta, Canada). Patients were assessed for eligibility and underwent a review of medical history, with emphasis on cardiovascular history and risk, as well as a review of lipid-lowering medication use at the screening visit. Baseline 12-h fasting measurements of LDL-C, total cholesterol (TC), triglycerides (TG) and high-density lipoprotein cholesterol (HDL-C) were conducted at local facilities within three days of the screening visit. The LDL-C was calculated using the standard Friedewald estimation method. However, when TG levels were > $3.99 \mathrm{mmol} / \mathrm{L}$, LDL-C was determined by ultracentrifugation since high TG levels have been shown to interfere with the accurate determination of LDL-C by the Friedewald equation [19]. Furthermore, direct enzymatic LDL-C assays which require less time and money have been shown to overestimate LDL-C when TG values are greater than $400 \mathrm{mg} / \mathrm{dl}$, leading to misclassification of the severity of dyslipidemia [20].

At the baseline visit, which took place within two weeks of the screening visit, patients were randomly assigned in a 1:1 ratio to either receive $10 \mathrm{mg} /$ day ezetimibe (Ezetrol ${ }^{\circledR}$, Merck) co-administered with their existing statin regimen (EZE + statin group), or double their current statin dose, for 6 weeks (STAT2 group). During randomized allocation the practitioner contacted an Interactive Voice Response System (IVRS) which issued a randomization number along with the treatment allocation. Randomization was centrally coordinated by a third-party data management center and was stratified by center. Patients allocated to the STAT2 group, who were on the highest possible dose of statin or were intolerant of higher statin doses as per the patient's or physician's assessment, were converted to the EZE + statin group. All patients were instructed not to change their diet or exercise habits during the study. There were no limitations on the type or dose of statin used at baseline. Ezetimibe was provided at no cost to the patient while statins were acquired via the existing insurance coverage. The final study visit took place 6 weeks after the baseline visit.

\section{Outcome measures}

The primary efficacy outcome measure was the percent change in plasma LDL-C concentration during the 6-week treatment period. Secondary efficacy measures included the changes in TC, TG, 
Table I. Demographics and baseline characteristics of patients

\begin{tabular}{|c|c|c|c|c|}
\hline \multirow[t]{2}{*}{ Characteristics } & \multicolumn{2}{|c|}{ Treatment group } & \multirow[t]{2}{*}{ Total $(n=936)$} & \multirow[t]{2}{*}{ Value of $p$} \\
\hline & $Z E+\operatorname{statin}(n=620)$ & STAT2 $(n=316)$ & & \\
\hline \multicolumn{5}{|l|}{ Age } \\
\hline Mean (SD) & $63(11.3)$ & $63(11.4)$ & $63(11.3)$ & \multirow{2}{*}{0.604} \\
\hline Range & $26-89$ & $28-87$ & $26-89$ & \\
\hline \multicolumn{5}{|l|}{ Age categories, $n$ (\%) } \\
\hline$\leq 45$ & $40(6.5)$ & $18(5.7)$ & $58(6.2)$ & \multirow{4}{*}{0.181} \\
\hline $46-65$ & $334(53.9)$ & $151(47.8)$ & $485(51.8)$ & \\
\hline $65-85$ & $237(38.2)$ & $144(45.6)$ & $381(40.7)$ & \\
\hline$\geq 85$ & $9(1.5)$ & $3(0.9)$ & $12(1.3)$ & \\
\hline \multicolumn{5}{|l|}{ Gender, $n(\%)$} \\
\hline Male & $392(63.2)$ & $191(60.4)$ & $583(62.3)$ & \multirow{2}{*}{0.406} \\
\hline Female & $228(36.8)$ & $125(39.6)$ & $353(37.7)$ & \\
\hline \multicolumn{5}{|l|}{ Co-morbidity and risk factor profile, $n$ (\%) } \\
\hline \multicolumn{5}{|l|}{ Smoking status } \\
\hline Current smoker & $130(21.0)$ & $68(21.5)$ & $198(21.2)$ & \multirow{3}{*}{0.611} \\
\hline Ex-smoker & $206(33.2)$ & $95(30.1)$ & $301(32.2)$ & \\
\hline Non-smoker & $284(45.8)$ & $153(48.4)$ & $437(46.7)$ & \\
\hline Hypertension & $345(55.6)$ & $179(56.6)$ & $524(56.0)$ & 0.809 \\
\hline Diabetes mellitus & $129(20.8)$ & $83(26.3)$ & $212(22.6)$ & 0.059 \\
\hline Metabolic syndrome & $82(13.2)$ & $24(7.6)$ & $106(11.3)$ & 0.010 \\
\hline Diabetes mellitus and metabolic syndrome & $174(28.1)$ & $91(28.8)$ & $265(28.3)$ & 0.464 \\
\hline Neither diabetes mellitus nor metabolic syndrome & me $235(37.9)$ & $118(37.3)$ & $353(37.7)$ & 0.867 \\
\hline Coronary artery disease & $262(42.3)$ & $129(40.8)$ & $391(41.8)$ & 0.674 \\
\hline Cerebrovascular disease & $73(11.8)$ & $35(11.1)$ & $108(11.5)$ & 0.752 \\
\hline Peripheral vascular disease & $60(9.7)$ & $26(8.2)$ & $86(9.2)$ & 0.468 \\
\hline Chronic kidney disease & $18(2.9)$ & $12(3.8)$ & $30(3.2)$ & 0.463 \\
\hline Family history of CVD & $300(48.4)$ & $163(51.6)$ & $463(49.5)$ & 0.418 \\
\hline \multicolumn{5}{|l|}{ Menopausal status $(n=353)$} \\
\hline Pre-menopause & $15(6.6)$ & $7(5.6)$ & $22(6.2)$ & \multirow{3}{*}{0.497} \\
\hline Peri-menopause & $7(3.1)$ & $4(3.2)$ & $11(3.1)$ & \\
\hline Post-menopause & $202(88.6)$ & $114(91.2)$ & $316(89.5)$ & \\
\hline Use of hormone replacement therapy & $37(16.2)$ & $17(13.6)$ & $54(15.3)$ & 0.486 \\
\hline
\end{tabular}

HDL-C and the TC/HDL-C ratio, as well as the proportion of patients achieving the recommended target LDL-C of $<2.5 \mathrm{mmol} / \mathrm{l}$ (2003 Canadian recommendations for the management of dyslipidemia and the prevention of cardiovascular disease [18]) or $<2.0 \mathrm{mmol} / \mathrm{l}$ (2009 Canadian guidelines for the diagnosis and treatment of dyslipidemia and prevention of cardiovascular disease [7]). Sample size requirements for the study were based on the primary efficacy measure. In order to detect a difference of $15 \%$ in the percent plasma LDL-C change between the two treatment groups with
$90 \%$ power and $5 \%$ significance, a sample size of 90 patients per group or 180 patients in total would be required. Similarly, for the subgroup analysis (diabetes and metabolic syndrome subgroup, diabetes subgroup, metabolic syndrome subgroup, and subgroup with neither of the two conditions) 180 patients per subgroup would be required. Based on the patients actually recruited in the current study (265 patients with diabetes and the metabolic syndrome, 212 patients with diabetes only, 106 patients with the metabolic syndrome only, and 353 patients with neither of the two 
Table II. Statin therapy at baseline*

\begin{tabular}{|c|c|c|c|c|c|c|c|c|c|}
\hline \multirow[t]{3}{*}{ Statin } & \multirow{3}{*}{$\begin{array}{c}\text { Patients, } \\
n(\%)^{\dagger}\end{array}$} & \multicolumn{8}{|c|}{ Total daily statin dose, $n$ (\%) } \\
\hline & & \multicolumn{2}{|c|}{$10 \mathrm{mg}$} & \multicolumn{2}{|c|}{$20 \mathrm{mg}$} & \multicolumn{2}{|c|}{$40 \mathrm{mg}$} & \multicolumn{2}{|c|}{$80 \mathrm{mg}$} \\
\hline & & EZE + statin & n STAT2 & EZE + statin & STAT2 & EZE + statin & STAT2 & $\mathrm{EZE}+$ statin & STAT2 \\
\hline Atorvastatin & $491(52.5)$ & $100(16.1)$ & $82(25.9)$ & 117 (18.9) & $67(21.2)$ & $75(12.1)$ & $24(7.6)$ & $15(2.4)$ & NA \\
\hline Simvastatin & $167(17.8)$ & $14(2.3)$ & $16(5.1)$ & $45(7.3)$ & $19(6.0)$ & $54(8.7)$ & $10(3.2)$ & $4(0.6)$ & NA \\
\hline Rosuvastatin & $162(17.3)$ & $71(11.5)$ & $39(12.3)$ & $25(4.0)$ & $10(3.2)$ & $7(1.1)$ & NA & NA & NA \\
\hline Pravastatin & $81(8.7)$ & $3(0.5)$ & $1(0.3)$ & $21(3.4)$ & $24(7.6)$ & $19(3.1)$ & $7(2.2)$ & $4(0.6)$ & NA \\
\hline Lovastatin & $27(2.9)$ & $2(0.3)$ & NA & $7(1.1)$ & $6(1.9)$ & $8(1.3)$ & $3(0.9)$ & $1(0.2)$ & NA \\
\hline Fluvastatin & $8(0.9)$ & NA & NA & $2(0.4)$ & $2(0.7)$ & $1(0.2)$ & $2(0.7)$ & $1(0.2)$ & NA \\
\hline Total & $936(100)$ & $190(30.6)$ & 138 (43.7) & $217(35.0) 1$ & $128(40.5)$ & $164(26.5)$ & 46 (14.6) & $25(4.0)$ & NA \\
\hline
\end{tabular}

${ }^{*}$ Before dose doubling, tPercentages are out of 936 patients enrolled: 620 in the EZE + statin group and 316 in the STAT2 group, §One patient reported taking atorvastatin $5 \mathrm{mg} /$ day, 3 patients were taking $30 \mathrm{mg} /$ day and 7 patients were taking $60 \mathrm{mg} /$ day; 1 patient each reported taking simvastatin $5 \mathrm{mg} /$ day, $30 \mathrm{mg} /$ day, $50 \mathrm{mg} /$ day, and 2 patients reported taking simvastatin $60 \mathrm{mg} /$ day; 8 patients reported taking rosuvastatin $5 \mathrm{mg} /$ day and 1 patient each reported taking $30 \mathrm{mg} /$ day and $60 \mathrm{mg} /$ day; 1 patient each reported taking a dose of $30 \mathrm{mg} /$ day and $60 \mathrm{mg} /$ day of pravastatin

conditions) the statistical power of the current study was $>0.99$ for the total population, the diabetes and metabolic syndrome subgroup, the diabetes subgroup and the subgroup with neither of the two conditions, while it was 0.63 for the metabolic subgroup.

Safety was assessed with clinical laboratory parameters and the incidence of treatmentemergent adverse events that were attributed to ezetimibe or the statin regimen according to the treating physician's judgment. All adverse events were reported according to the MedDRA dictionary of terms, version 9 [21].

\section{Statistical analysis}

The intention-to-treat (ITT) principle, including all patients who received at least one dose of ezetimibe or double statin dose, was employed to analyze the study outcomes. All analyses were conducted for the total patient population, patients with diabetes and the metabolic syndrome (as defined by the 2005 modified International Diabetes Federation criteria [22]), patients with diabetes only, patients with the metabolic syndrome only, and patients with neither of the two conditions, using the SPSS software, version 12.0. Statistical significance for between-group differences in continuous outcomes was assessed with the twotailed Student's $t$-test for independent samples, while the non-parametric Mann-Whitney test was used for patient subgroups. Multiple linear regression and general linear models (GLM) were used to assess between-group differences with respect to the primary efficacy measure (dependent variable: \% change in LDL-C), adjusting for potential confounders (independent variables: treatment group, baseline statin dose).

\section{Results}

\section{Baseline characteristics}

A total of 1,155 patients were screened between May 2005 and June 2006, among whom 936 underwent randomization. Of these patients, 459 (49.0\%) were initially randomized to the EZE + statin group and $477(51.0 \%)$ to the STAT2 group. A total of 161 patients $(33.8 \%)$ in the STAT2 group were converted to the EZE + statin group: 150 (93.2\%) because of patient or physician concerns about the tolerance of higher statin doses, and 11 (6.8\%) because they were at the maximum statin dose. Therefore, the final treatment allocation was 620 patients $(66.2 \%)$ in the EZE + statin group and 316 patients $(33.8 \%)$ in the STAT2 group. No differences with respect to baseline characteristics between the STAT2 patients who were converted to the EZE + statin group and those who were maintained in the STAT2 group were observed. There were 51 patients (5.4\%), 34 (5.5\%) in the EZE + statin group and $17(5.4 \%)$ in the STAT2 group, who were withdrawn prior to the 6-week final assessment. Reasons for withdrawal were as follows: loss to follow-up for 30 (3.2\%), adverse events for 15 (1.6\%), withdrawal of consent for $5(0.5 \%)$, while one patient in the EZE + statin group was withdrawn due to intolerance to the statin.

As summarized in Table I, demographics and baseline clinical characteristics for the ITT population exhibited no significant differences between groups. The mean (SD) age of the study sample was $63(11.3)$ years while $62.3 \%$ were male. A total of 859 patients (91.8\%) were classified as being at high ( $\geq 20 \%$ ) 10-year risk for CAD on the basis of confirmed diabetes or atherosclerotic disease and the remaining 77 (8.2\%) were classified 


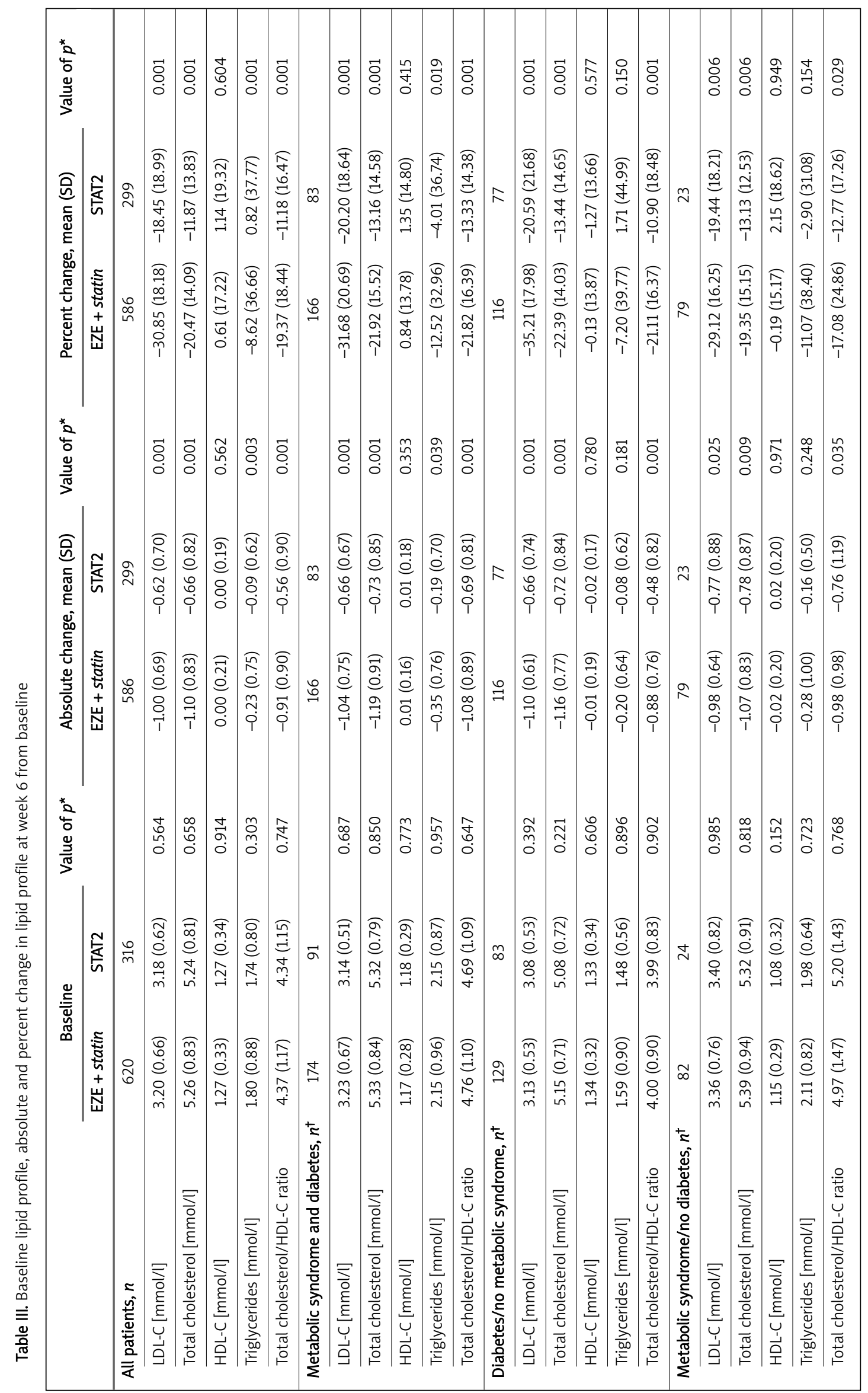




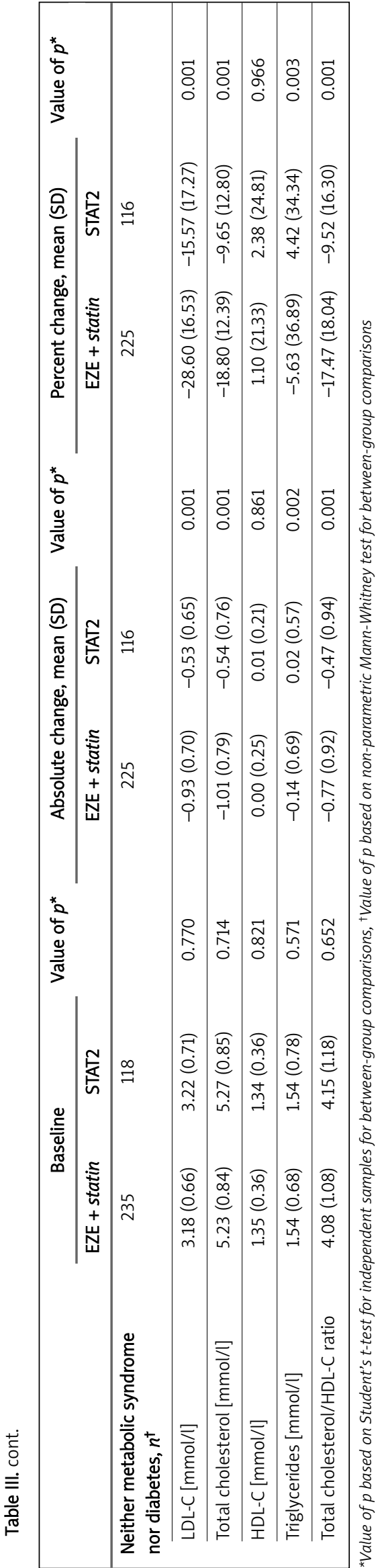

as high risk on the basis of the Framingham model. There were 212 patients (22.6\%) with diabetes but not the metabolic syndrome, 106 (11.3\%) with the metabolic syndrome but not diabetes, 265 (28.3\%) with both diabetes mellitus and the metabolic syndrome and $353(37.7 \%)$ with neither diabetes nor the metabolic syndrome. The profile of statin therapy at baseline is shown in Table II, with more than half (52.5\%) of the study subjects taking atorvastatin, followed by simvastatin $(17.8 \%)$, rosuvastatin (17.3\%) and pravastatin (8.7\%).

\section{Lipid profile}

After 6 weeks of treatment, patients in the EZE + statin group experienced a significantly larger reduction in LDL-C (-30.8\% vs. $-18.4 \%$ in EZE + statin and STAT2, respectively), TC $(-20.5 \%$ vs. $-11.9 \%$ in EZE + statin and STAT2, respectively), TG (-8.6\% vs. $-0.8 \%$ in EZE + statin and STAT2, respectively) and TC/HDL-C ratio ( $-19.4 \%$ vs. $-11.2 \%$ in EZE + statin and STAT2, respectively) (Table III). Changes in HDL-C, however, were not significantly different between the two groups ( $0.6 \%$ vs. $1.1 \%$ in EZE + statin and STAT2, respectively). These differences were observed for both the total study sample and all patient subgroups. Similar results were observed for the subgroup of patients who converted to the EZE + statin group (data not shown).

Multivariate linear regression analysis was further used to adjust the between-group differences with respect to the percent change in LDL-C during the six-week treatment period for the effect of the baseline statin dose. This analysis indicated that co-administration of ezetimibe had a significant effect $(p<0.001)$ on reducing LDL-C by an additional $19.7 \%$ (SE: 1.36) over and above the statin effect (Table IVA). The results also showed that for every doubling of the baseline statin dose used, the additional decrease in LDL-C is 5.4\% (SE: 0.52). Table IVB summarizes the least-squares mean estimates of the adjusted mean percent reduction in LDL-C for the two treatment groups by baseline statin dose. These results show that for the sample as a whole the adjusted mean (SD) percent decrease in LDL-C after co-administration of ezetimibe with the current statin was 30.8\% (4.95) compared to a mean (SD) decrease of 9.3\% (3.87) for doubling the statin dose $(p<0.001)$. The difference was consistent across all statin doses.

After 6 weeks of treatment, $70 \%$ of patients in the EZE + statin group and $48 \%$ of patients in the STAT2 group who completed the study achieved target LDL-C levels of less than $2.5 \mathrm{mmol} / \mathrm{l}$ (odds ratio [OR] 2.45, 95\% $\mathrm{Cl} 1.85-3.24, p<0.001$ ). Similarly, a significantly higher proportion of patients in the EZE + statin group (40\%) compared with the STAT2 group (18\%) achieved final serum LDL-C levels less than $2.0 \mathrm{mmol} / \mathrm{l}(\mathrm{OR} 2.97,95 \% \mathrm{Cl}$ 
2.14-4.13, $p<0.001)$. Similar results were observed for all four patient subgroups analyzed (Figure 1).

\section{Safety}

The incidence and profile of adverse events between the study groups were in general similar between the two groups, with the exception of a higher incidence of myalgia and muscle spasms in patients in the STAT2 group (2.8\% and $0.9 \%$ of patients, respectively) compared with the EZE + statin group ( $0.8 \%$ and $0 \%$ of patients, respectively). A total of 62 non-serious adverse events (NSAEs) were reported by 42 patients $(6.8 \%)$ in the EZE + statin group. Of these, $16(25.8 \%)$ NSAEs were reported by 16 (9.9\%) of the 161 patients who were converted from the STAT2 to the EZE + statin group. There were 22 NSAEs reported by 18 patients (5.7\%) in the STAT2 group. The majority (97.6\%) of the treatment-emergent NSAEs were mild or moderate in severity. Two $(2.4 \%)$ treatment-emergent NSAEs of severe intensity, diarrhoea and abdominal pain, were reported by one patient in the EZE + statin group. Two patients in the EZE + statin group (1 randomized and 1 converted) experienced nonspecified increases in hepatic enzymes. No serious adverse events were reported in this trial.

\section{Discussion}

Although statin treatment is the predominant lipid-lowering strategy, a significant proportion of patients with hypercholesterolemia do not achieve target LDL-C levels on statin monotherapy [4-6]. Combination of ezetimibe with low-dose statin has been shown to be effective in improving the lipid profile in the context of controlled clinical trials, providing an additional $20 \%$ to $25 \%$ reduction in low density lipoprotein cholesterol (LDL-C) compared with statin monotherapy $[23,24]$. In this
Table IVA. Multivariate linear regression analysis results

\begin{tabular}{|lccc|}
\hline Variable & \multicolumn{2}{c}{ Coefficient } & Value of $p$ \\
\cline { 2 - 3 } & Estimate & SE & \\
\hline Group $^{\star}$ & -19.66 & 1.36 & $<0.001$ \\
\hline Baseline statin dose $^{\dagger}$ & -5.41 & 0.52 & $<0.001$ \\
\hline
\end{tabular}

Table IVB. Least-squares mean estimates of percent change in LDL-C

\begin{tabular}{|lccc|}
\hline \multirow{2}{*}{$\begin{array}{l}\text { Baseline statin } \\
\text { dose }[\mathrm{mg} / \text { day }]\end{array}$} & \multicolumn{3}{c|}{ Treatment group, LS mean (SD) } \\
\cline { 2 - 4 } & EZE + statin & STAT2 & All patients \\
\hline $10^{\ddagger}$ & $-25.0(0.05)$ & $-5.4(0.01)$ & $-16.9(9.69)$ \\
\hline $20^{£}$ & $-30.5(0.03)$ & $-10.8(0.05)$ & $-23.2(9.52)$ \\
\hline 40 & $-35.9(0.05)$ & $-16.2(0.11)$ & $-31.3(8.37)$ \\
\hline $80^{€}$ & $-41.4(0.05)$ & NA & $-41.4(0.05)$ \\
\hline Total & $-30.8(4.95)$ & $-9.3(3.87)$ & $-23.5(11.22)$ \\
\hline
\end{tabular}

Multivariate linear regression dependent variable: percent change in LDL-C during 6-week treatment period; independent variables: treatment group, baseline statin dose, SE - standard error, LS meanleast-squares mean, *Group $-E Z E+$ statin $=1$, STAT2 $=0$, ${ }^{*}$ Baseline statin dose: $10 \mathrm{mg} /$ day $=1,20 \mathrm{mg} /$ day $=2,40 \mathrm{mg} /$ day $=3,80 \mathrm{mg} /$ day $=4$, $¥$ Includes 10 patients who reported taking a statin dose of $5 \mathrm{mg} /$ day, IIncludes 6 patients who reported taking a statin dose of $30 \mathrm{mg} /$ day, $€ /$ ncludes 12 patients who reported taking a statin dose of $50 \mathrm{mg} /$ day or $60 \mathrm{mg} /$ day

study we compared the efficacy and safety of ezetimibe added to the existing statin regimen with that of doubling the dose of the statin, in a real-life setting; patients were recruited from physicians' practices regardless of their treating statin and they continued to receive their existing statin, either in combination with ezetimibe or at a double dose, throughout the course of the study.

The results of the EZE(STAT) ${ }^{2}$ study indicate that co-administration of ezetimibe $10 \mathrm{mg} /$ day with any statin regimen is more effective in reducing serum

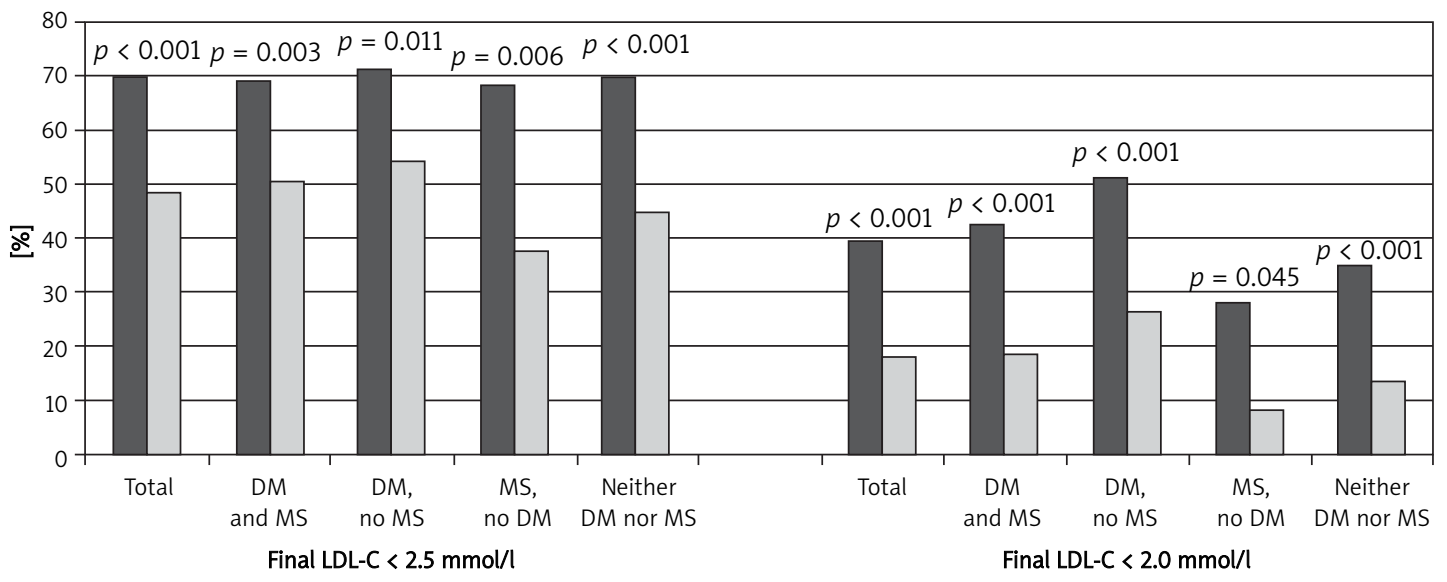

$\square \mathrm{EZE}+$ statin $\quad \square \mathrm{STAT2}$

Figure 1. Final LDL-C targets by treatment group

$D M$ - diabetes mellitus, MS - metabolic syndrome 
LDL-C compared to doubling of the statin dose (change in LDL-C levels: $30.9 \%$ vs. $18.5 \%$ in the EZE + statin and STAT2 group, respectively, or $30.8 \%$ vs. $9.3 \%$, respectively, when adjusting for the statin dose; \% of patients achieving LDL-C $<2.5 \mathrm{mmol} / \mathrm{l}$ : $70 \%$ vs. $48 \%$, respectively). Moreover, significantly more patients in the EZE + statin group achieved the LDL-C targets of $<2.5$ and $<2.0 \mathrm{mmol} / \mathrm{l}$ currently recommended by the European Society of Cardiology (ESC) and the US treatment guidelines, or the Canadian Cardiovascular Society, respectively $[7,25,26]$. In addition, co-administration of ezetimibe with statins produced a more profound beneficial effect on the patient's overall lipid profile (TC, TG, TC/HDL-C ratio) compared with doubling the statin dose. The above-mentioned differences were observed across patient subgroups with diabetes and/or the metabolic syndrome that are at increased risk for cardiovascular disease. Our results are in agreement with recent studies showing a similar lipid-lowering beneficial effect of co-administering ezetimibe with atorvastatin $[9,10$, 14] and simvastatin [11-13].

Ezetimibe co-administered with statin was generally well tolerated, with a similar safety profile and incidence of adverse events to the statin treatment, again in agreement with recent studies [9-14]. The only exception was a higher incidence of myalgia and muscle spasms observed in the STAT2 group, an effect that has been previously associated with high doses of statins [16, 17].

Reduction of serum LDL-C levels is the primary factor in preventing coronary heart disease [27]. In addition to their cholesterol-reducing function, statins exert additional pleiotropic effects such as improving endothelial dysfunction, thrombosis and vascular inflammation [28]. Our study was not designed to define the effect of ezetimibe on these parameters. However, recent studies indicate that ezetimibe may also have similar pleiotropic effects [29-32] and additional trials are ongoing which will further examine the impact of ezetimibe on these effects [33-35].

In conclusion, our study demonstrates that, in patients at high risk for coronary artery disease who have not achieved the LDL-C target with statin monotherapy, co-administration of ezetimibe is a well-tolerated and more effective strategy in improving the lipid profile compared with doubling the existing statin dose.

\section{References}

1. World Health Organization (2004). "Annex Table 2: Deaths by cause, sex and mortality stratum in WHO regions, estimates for 2002". Retrieved 01-25-2010.

2. Smith SC Jr, Allen J, Blair SN, et al. AHA/ACC guidelines for secondary prevention for patients with coronary and other atherosclerotic vascular disease: 2006 update endorsed by the National Heart, Lung, and Blood Institute. J Am Coll Cardiol 2006; 47: 2130-9.

3. Yusuf S, Hawken S, Ounpuu S, et al. Effect of potentially modifiable risk factors associated with myocardial infarction in 52 countries (the INTERHEART study): casecontrol study. Lancet 2004; 364: 937-52.

4. Bourgault C, Davignon J, Fodor G, et al. Statin therapy in Canadian patients with hypercholesterolemia: the Canadian Lipid Study-Observational (CALIPSO). Can J Cardiol 2005; 21: 1187-93.

5. Foley KA, Simpson RJ Jr, Crouse JR III, Weiss TW, Markson LE, Alexander CM. Effectiveness of statin titration on lowdensity lipoprotein cholesterol goal attainment in patients at high risk of atherogenic events. Am J Cardiol 2003; 92: 79-81.

6. Pearson TA, Laurora I, Chu H, Kafonek S. The lipid treatment assessment project (L-TAP): a multicenter survey to evaluate the percentages of dyslipidemic patients receiving lipid-lowering therapy and achieving low-density lipoprotein cholesterol goals. Arch Intern Med 2000; 160: 459-67.

7. Genest J, McPherson R, Frohlich J, et al. 2009 Canadian Cardiovascular Society/Canadian guidelines for the diagnosis and treatment of dyslipidemia and prevention of cardiovascular disease in the adult - 2009 recommendations. Can J Cardiol 2009; 25: 567-79.

8. Sudhop T, Lutjohann D, Kodal A, et al. Inhibition of intestinal cholesterol absorption by ezetimibe in humans. Circulation 2002; 106: 1943-8.

9. Conard SE, Bays HE, Leiter LA, et al. Efficacy and safety of ezetimibe added on to atorvastatin $(20 \mathrm{mg})$ versus uptitration of atorvastatin (to $40 \mathrm{mg}$ ) in hypercholesterolemic patients at moderately high risk for coronary heart disease. Am J Cardiol 2008; 102: 1489-94.

10. Leiter LA, Bays H, Conard S, et al. Efficacy and safety of ezetimibe added on to atorvastatin $(40 \mathrm{mg}$ ) compared with uptitration of atorvastatin (to $80 \mathrm{mg}$ ) in hypercholesterolemic patients at high risk of coronary heart disease. Am J Cardiol 2008; 102: 1495-501.

11. Roeters van Lennep HW, Liem AH, Dunselman PH, LingaThie GM, Zwinderman AH, Jukema JW. The efficacy of statin monotherapy uptitration versus switching to ezetimibe/simvastatin: results of the EASEGO study. Curr Med Res Opin 2008; 24: 685-94.

12. Farnier M, Averna M, Missault L, et al. Lipid-altering efficacy of ezetimibe/simvastatin 10/20 mg compared with rosuvastatin $10 \mathrm{mg}$ in high-risk hypercholesterolaemic patients inadequately controlled with prior statin monotherapy - The IN-CROSS study. Int J Clin Pract 2009; 63: 547-59.

13. Feldman T, Koren M, Insull W Jr, et al.Treatment of highrisk patients with ezetimibe plus simvastatin coadministration versus simvastatin alone to attain National Cholesterol Education Program Adult Treatment Panel III low-density lipoprotein cholesterol goals. Am J Cardiol 2004; 93: 1481-6.

14. Stein E, Stender S, Mata P, et al. Achieving lipoprotein goals in patients at high risk with severe hypercholesterolemia: efficacy and safety of ezetimibe co-administered with atorvastatin. Am Heart J 2004; 148: 447-55.

15. Ostad MA, Eggeling S, Tschentscher P, et al. Flowmediated dilation in patients with coronary artery disease is enhanced by high dose atorvastatin compared to combined low dose atorvastatin and ezetimibe: results of the CEZAR study. Atherosclerosis 2009; 205: 227-32.

16. Hedenmalm K, Alvan G, Ohagen P, Dahl ML. Muscle toxicity with statins. Pharmacoepidemiol Drug Saf 2010; 19: 223-31. 
17. Backes JM, Howard PA, Ruisinger JF, Moriarty PM. Does simvastatin cause more myotoxicity compared with other statins? Ann Pharmacother 2009; 43: 2012-20.

18. Genest J, Frohlich J, Fodor G, McPherson R. Recommendations for the management of dyslipidemia and the prevention of cardiovascular disease: summary of the 2003 update. CMAJ 2003; 169: 921-4.

19. Friedewald WT, Levy RI, Fredrickson DS. Estimation of the concentration of low-density lipoprotein cholesterol in plasma, without use of the preparative ultracentrifuge. Clin Chem 1972; 18: 499-502.

20. Evans SR, Fichtenbaum CJ, Aberg JA; A5087 Study Team. Comparison of direct and indirect measurement of LDL-C in HIV-infected individuals: ACTG 5087. HIV Clin Trials 2007; 8: 45-52.

21. International Federation of Pharmaceutical Manufacturers Associations. Medical Dictionary for Regulatory Activities (MedDRA) Version 9.0. Chantilly, VA: MedDRA MSSO. 2007. Ref type: Catalog.

22. International Diabetes Federation: The IDF consensus worldwide definition of the metabolic syndrome [article online], 2005. Available at: http://www.idf.org/webdata/ docs/MetSyndrome_FINAL.pdf. Accessed on 11 December 2009. Ref type: Generic.

23. Mikhailidis DP, Sibbring GC, Ballantyne CM, Davies GM, Catapano AL. Meta-analysis of the cholesterol-lowering effect of ezetimibe added to ongoing statin therapy. Curr Med Res Opin 2007; 23: 2009-26.

24. Angelopoulos J, Krassakopoulos N, Nathanson R, Boukas $\mathrm{S}$, Sampalis JS. Co-administration of ezetimibe and a statin in management of dyslipidemias: a meta-analysis of clinical trials. Arch Med Sci 2009; 5: 347-63.

25. Grundy SM, Cleeman JI, Merz CN, et al. Implications of recent clinical trials for the National Cholesterol Education Program Adult Treatment Panel III Guidelines. J Am Coll Cardiol 2004; 44: 720-32.

26. Graham I, Atar D, Borch-Johnsen K, et al. European guidelines on cardiovascular disease prevention in clinical practice: executive summary. Fourth Joint Task Force of the European Society of Cardiology and other societies on cardiovascular disease prevention in clinical practice (constituted by representatives of nine societies and by invited experts). Eur J Cardiovasc Prev Rehabil 2007; 14 Suppl 2: E1-40.

27. Baigent C, Keech A, Kearney PM, et al. Efficacy and safety of cholesterol-lowering treatment: prospective metaanalysis of data from 90,056 participants in 14 randomised trials of statins. Lancet 2005; 366: 1267-78.

28. Davignon J. Beneficial cardiovascular pleiotropic effects of statins. Circulation 2004; 109 (23 Suppl 1): III39-43.

29. Yamaoka-Tojo M, Tojo T, Kosugi R, et al. Effects of ezetimibe add-on therapy for high-risk patients with dyslipidemia. Lipids Health Dis 2009; 8: 41.

30. Araujo DB, Bertolami MC, Ferreira WP, et al. Pleiotropic effects with equivalent low-density lipoprotein cholesterol reduction: comparative study between simvastatin and simvastatin/ezetimibe coadministration. J Cardiovasc Pharmacol 2010; 55: 1-5.

31. Kalogirou M, Tsimihodimos V, Elisaf M. Pleiotropic effects of ezetimibe: do they really exist? Eur J Pharmacol 2010; 633: 62-70.

32. Kater AL, Batista MC, Ferreira SR. Synergistic effect of simvastatin and ezetimibe on lipid and pro-inflammatory profiles in pre-diabetic subjects. Diabetol Metab Syndr 2010; 2 : 34.
33. Baigent C, Landry M. Study of Heart and Renal Protection (SHARP). Kidney Int Suppl 2003; 84: S207-S210.

34. Devine PJ, Turco MA, Taylor AJ. Design and rationale of the ARBITER 6 trial (Arterial Biology for the Investigation of the Treatment Effects of Reducing Cholesterol)-6-HDL and LDL Treatment Strategies in Atherosclerosis (HALTS). Cardiovasc Drugs Ther 2007; 21: 221-5.

35. Cannon CP, Giugliano RP, Blazing MA, et al. Rationale and design of IMPROVE-IT (IMProved Reduction of Outcomes: Vytorin Efficacy International Trial): comparison of ezetimbe/simvastatin versus simvastatin monotherapy on cardiovascular outcomes in patients with acute coronary syndromes. Am Heart J 2008; 156: 826-32. 